\title{
Tip dating with fossil sites and stratigraphic sequences
}

\author{
Benedict King ${ }^{\text {Corresp., } 1,}$, Martin Rücklin ${ }^{1}$ \\ ${ }^{1}$ Naturalis Biodiversity Center, Leiden, Netherlands \\ Corresponding Author: Benedict King \\ Email address: benedict.king@naturalis.nl
}

Tip dating, a method of phylogenetic analysis in which fossils are included as terminals and assigned an age, is becoming increasingly widely used in evolutionary studies. Current implementations of tip dating allow fossil ages to be assigned as a point estimate, or incorporate uncertainty through the use of uniform tip age priors. However, the use of tip age priors has the unwanted effect of decoupling the ages of fossils from the same fossil site. Here we introduce a new Markov Chain Monte Carlo (MCMC) proposal, which allows fossils from the same site to have linked ages, while still incorporating uncertainty in the age of the fossil site itself. We also include an extension, allowing fossil sites to be ordered in a stratigraphic column with age bounds applied only to the top and bottom of the sequence. These MCMC proposals are implemented in a new open-source BEAST2 package, palaeo. We test these new proposals on a dataset of early vertebrate fossils, concentrating on the effects on two sites with multiple acanthodian fossil taxa but wide age uncertainty, the Man On The Hill (MOTH) site from northern Canada, and the Turin Hill site from Scotland, both of Lochkovian (Early Devonian) age. The results show an increased precision of age estimates when fossils have linked tip ages compared to when ages are unlinked, and in this example leads to support for a younger age for the MOTH site compared with the Turin Hill site. There is also a minor effect on the tree topology of acanthodians. These new MCMC proposals should be widely applicable to studies that employ tip dating, particularly when the terminals are coded as individual specimens. 


\section{Tip dating with fossil sites and stratigraphic}

\section{2 sequences}

3

4 Benedict King ${ }^{1}$, Martin Rücklin ${ }^{1}$

5

$6 \quad{ }^{1}$ Endless Forms Group, Naturalis Biodiversity Center, Leiden, Zuid-Holland, The Netherlands

7

8 Corresponding author:

9

10 Benedict King ${ }^{1}$

11

12 Email address: benedict.king@naturalis.nl 


\section{Abstract}

14 Tip dating, a method of phylogenetic analysis in which fossils are included as terminals and assigned an age, is becoming increasingly widely used in evolutionary studies. Current implementations of tip dating allow fossil ages to be assigned as a point estimate, or incorporate uncertainty through the use of uniform tip age priors. However, the use of tip age priors has the unwanted effect of decoupling the ages of fossils from the same fossil site. Here we introduce a new Markov Chain Monte Carlo (MCMC) proposal, which allows fossils from the same site to have linked ages, while still incorporating uncertainty in the age of the fossil site itself. We also include an extension, allowing fossil sites to be ordered in a stratigraphic column with age bounds applied only to the top and bottom of the sequence. These MCMC proposals are implemented in a new open-source BEAST2 package, palaeo. We test these new proposals on a dataset of early vertebrate fossils, concentrating on the effects on two sites with multiple acanthodian fossil taxa but wide age uncertainty, the Man On The Hill (MOTH) site from northern Canada, and the Turin Hill site from Scotland, both of Lochkovian (Early Devonian) age. The results show an increased precision of age estimates when fossils have linked tip ages compared to when ages are unlinked, and in this example leads to support for a younger age for the MOTH site compared with the Turin Hill site. There is also a minor effect on the tree topology of acanthodians. These new MCMC proposals should be widely applicable to studies that employ tip dating, particularly when the terminals are coded as individual specimens.

\section{Introduction}

Tip dating is increasingly used as a method to calibrate molecular phylogenies and to analyse phylogenies with fossil taxa (Gavryushkina et al. 2017; Lee et al. 2014; Ronquist et al. 2012). Central to tip dating are the ages given to the individual fossil taxa (or tips). Often this has been in the form of point estimates, but usually there is some uncertainty regarding the precise age of a fossil. Simulations have shown that better performance is achieved when each fossil is given a uniform tip age prior across the range of uncertainty (Barido-Sottani et al. 2019).

Current implementations of tip dating only allow tip ages to vary independently from each other. This has the undesired effect of separating the ages of fossil taxa from the same site. In reality, it is frequently the case that a fossil site has a wide uncertainty regarding age, but it is known that all the fossils from that site are of approximately the same age. A striking example, from the empirical dataset used in this study, is the so-called "wonder block" from the Man On The Hill (MOTH) site from the Lochkovian (419.2-410.8 million years) of northern Canada (Hanke \& Wilson 2006). This single block contains the acanthodians Obtusacanthus, Brochoadmones and Lupopsyrus (Hanke \& Wilson 2006), but these fossils can be separated by millions of years in a tip-dated analysis in which age uncertainty is dealt with in the typical manner (King et al. 2017).

A second limitation is that a series of fossil sites can often be placed in chronological order, despite the upper and lower bounds for the age uncertainty of these sites overlapping. For 
53 example, radiometric dates might be known only for the top and bottom of a geological

54 formation, and fossils known from several layers within this formation. Therefore, although the

55 total range of uncertainty for the age of each fossiliferous layer is the same, it is known in which

56 order the layers occur. In current implementations of tip dating, it would be necessary to either

57 impose arbitrary age bounds to maintain the chronological order of the layers, or to allow the

58 layers to be sampled in the incorrect order.

59

60

61

62

63

64

65

66

67

68

69

70

71

72

73

74

75

76

77

78

79

80

81

82

83

84

85

86

87

88

89

90

91

92

In this study we introduce new MCMC proposals for the software BEAST2 (Bouckaert et al. 2019), which allow linking of tip ages for fossils from the same site, as well as the ordering of fossil sites within a stratigraphic sequence. We test these proposals on a dataset of early gnathostome fossils (King et al. 2017), focusing on two fossil sites with multiple taxa but wide age uncertainty ranges: the Man On The Hill (MOTH) site from the Lochkovian (Early Devonian) of Canada, and the Turin Hill (or Tillywhandland) site from the Lochkovian of Scotland.

\section{Materials \& Methods}

Tip date MCMC proposals for fossil sites are implemented with new operators in BEAST2, available in the BEAST2 package palaeo (available for download at https://github.com/kingben/palaeo). The package includes an $\mathrm{R}$ function that generates $\mathrm{xml}$ code for these operators from tables of fossil site occurrences.

The first operator, FossilSiteDateRandomWalker, an extension of SampledNodeDateRandom Walker from the sampled ancestors package (Gavryushkina et al. 2014), takes as input the list of taxa and the age bounds for the site. New proposals, consisting of a random age within the upper and lower bounds, are applied to all taxa in the site simultaneously. Each fossil site requires a separate operator. A second operator, RelativeFossilSiteDateRandomWalker, allows the ordering of sites within a stratigraphic sequence, while allowing overlapping upper and lower bounds. The additional inputs are fossil sites that sit above and/or below within the sequence. This operator effectively implements a prior on the relative ages of fossil layers. New proposals for fossil site ages that fall outside bounds defined by the age estimates for the sites occurring immediately below or above within a sequence are immediately rejected (i.e. assigned a prior probability of 0 ). The acceptable bounds for the age estimates of sites therefore depend on the other sites in the sequence, and will change as the MCMC chain runs (Fig. 1A-B).

We tested the performance of these new MCMC proposals on the dataset of King et al. (2017), a dataset that includes autapomorphies, which can be important for tip dating (Matzke \& Irmis 2018). We updated the BEAST2 $\mathrm{xml}$ files, first with independent age priors for each fossil, using the SampledNodeDateRandomWalker operator from the sampled ancestors package

(Gavryushkina et al. 2014), and second with the new MCMC proposals described here. Analyses 
93

94

95

96

97

98

99

100

101

102

103

104

105

106

107

108

109

110

111

112

113

114

115

116

117

118

119

120

121

122

123

124

125

126

127

128

129

130

131

132

used an uncorrelated lognormal clock (Drummond et al. 2006). The prior on clock rate was an exponential with mean 0.003 and offset 0.0016 , while the prior on clock standard deviation was an exponential with mean 1. We used the Mkv model (Lewis 2001) and gamma distributed among-character rate variation (Yang 1996) with four rate categories; the prior on the shape parameter was a uniform distribution on the range $0-10$. The tree prior was a sampled ancestor birth-death model (Gavryushkina et al. 2014), with a lognormal distribution prior (mean in real space 0.14 , standard deviation 0.9 ) on birth rate, an exponential prior (mean 0.1) on death rate and an exponential prior (mean 0.03 ) on sampling rate. The analyses were run for 200,000,000 generations across four independent runs, with a 10\% burn-in, and convergence was confirmed using Tracer (Rambaut et al. 2014) and Rwty (Warren et al. 2017). To test the effect of using a RelativeFossilSiteDateRandomWalker operator, we also ran an analysis sampling from the prior only.

Post-processing of results was performed in R (R Core Team 2018), utilising the packages ape (Paradis et al. 2004), phytools (Revell 2012) and ggplot2 (Wickham 2016). Following the recommendations for summarising trees in O'Reilly \& Donoghue (2018), 50\% majority-rule consensus trees were calculated in the R package ape (Paradis et al. 2004) and posterior probabilities were calculated for nodes on this tree in TreeAnnotator 1.10.2 (Suchard et al. 2018). We focused attention on two sites in particular, the Man On The Hill site (MOTH) and Turin Hill. Both have wide age uncertainty (Lochkovian, 419.2-410.8 Million years), but contain several taxa: eight and five acanthodians respectively for this particular analysis. Full results and analysis scripts are available on the github repository (https://github.com/king-ben/palaeo).

\section{Results}

We demonstrate the effect of the RelativeFossilSiteDateRandomWalker on taxa from the Devonian Red Bay Group from Spitzbergen, specifically taxa from the older Fraenkelryggen and younger Ben Nevis formations (Fig. 1C). As expected, implementation of this operator led to a non-uniform effective prior on tip ages (Fig. 1C). The effective prior on age of the Fraenkelryggen Formation taxa was concentrated in the older part of the age uncertainty range, while the converse was true for the Ben Nevis Formation taxa. However, values across the entire span were sampled for both.

When estimated independently (i.e. with SampledNodeDateRandomWalker), individual taxa from the same fossil site could show widely variable dates within a single tree from the posterior sample. Across the posterior sample, the age estimates for taxa from the Turin Hill site were spread over an average range of 5.43 million years, while the range for MOTH was 6.55 million years.

The FossilSiteDateRandomWalker operator resulted in increased precision of site age estimates when compared with estimates for each taxon independently (Fig. 2A). The 95\% highest 
133 posterior density (HPD) interval spanned 4.79 million years for the MOTH site, whereas the 134 HPD intervals for individual MOTH taxa when given independent ages spanned between 5.53 135 and 7.92 million years. The HPD interval for the Turin hill site spanned 6.08 million years,

136

137

138

139

140

141

142

143

144

145

146

147

148

149

150

151

152

153

154

155

156

157

158

159

160

161

162

163

164

165

166

167

168

169

170

171

compared with between 7.36 and 7.77 million years for the individual taxa.

The analysis with linked ages within each fossil site supported a younger age for the MOTH site compared to Turin Hill. The median age for MOTH was 412.36 million years (HPD 410.80415.59), whereas the median for Turin Hill was 416.47 million years (HPD 413.06-419.15). MOTH was younger than Turin Hill in $97 \%$ of trees from the posterior sample.

Some support a younger age for MOTH than Turin Hill was also present in the analysis with independent dates, although the effect was less strong. The age estimate for the two sites in each sample from the posterior was calculated as the mean of the age estimates for the individual taxa. The median across the posterior sample of this estimate was 414.57 for MOTH and 415.40 for Turin Hill. The mean age for MOTH taxa was younger than the mean for Turin Hill taxa in $76 \%$ of trees from the posterior sample. Therefore, use of linked tip dates amplifies support for a younger age for MOTH. Notably the median age estimate for the MOTH site when tip ages were linked was younger than the median age estimate for any of the individual MOTH taxa when tip ages were independent (Fig. 2A). Conversely, the median age estimate for Turin Hill was older than the estimates for any of its individual taxa (Fig. 2A).

Use of linked tip dates had a minor effect on tree topology (Fig. 2B-C). The 50\% majority-rule consensus tree for the analysis with independent tip dates showed the MOTH taxon Cassidiceps resolved as the sister group to a clade consisting of Mesacanthus, Promesacanthus, Cheiracanthus, Homalacanthus and Acanthodes (Fig. 2B). When tip dates within fossil sites are linked, this node collapses into a polytomy (Fig. 2C).

\section{Discussion}

The results show that linking tip ages from fossil sites can lead to an increase in the precision of age estimates when compared with analyses allowing independent tip dates. This may have important implications for the use of Bayesian phylogenetic estimation of fossil ages (Drummond \& Stadler 2016), in cases where a fossil site has uncertain dates but multiple taxa. For example, in an analysis estimating the age of fossil sites containing phiomorph rodent fossils, the estimated ages for fossils within a single site were sometimes different (Sallam \& Seiffert 2016). The use of linked tip dates should therefore increase the accuracy and precision of such estimates. In theory, further extensions to these tip date operators could even allow the use of multiple trees (i.e. several groups analysed simultaneously), with dates for fossil sites linked across the trees, to further increase precision. 
172 The age estimates for the MOTH and Turin Hill sites should for now be treated with caution. The 173 younger age estimate for the MOTH site is likely driven by the similarities of some taxa with 174 chondrichthyans (Hanke \& Wilson 2004; Hanke \& Wilson 2010), and others with diplacanthid 175 acanthodians (Hanke \& Davis 2008; Hanke et al. 2001). The earliest chondrichthyan fossil for 176 which good morphological data is known is Doliodus (Miller et al. 2003), of Emsian (late Early 177 Devonian) or early Eifelian (early Middle Devonian) age. Diplacanthid acanthodians are mainly 178 found in the Middle Devonian (Burrow et al. 2016). However, the presumed poor sampling of 179 early chondrichthyans in the fossil record (Coates et al. 2018), combined with the sparsity of 180 morphological characters that can be coded for even the best-preserved acanthodian fossils, 181 means that this result should be considered preliminary.

182

183

184

185

186

187

188

189

190

191

192

193

194

195

196

197

198

199

200

201

202

203

204

205

206

207

208

209

210

211

Linking tip dates can affect the phylogenetic position of fossils. The affected taxon, Cassidiceps, from the MOTH site, has the oldest age estimate of the MOTH acanthodians when tip dates are allowed to vary independently (Fig. 2A). Enforcing all MOTH taxa to have the same age therefore leads to a larger difference in the sampled age for Cassidiceps, leading to increased sampling in a more nested position (Fig. 2C). Relative to other MOTH acanthodians, the morphology of Cassidiceps is relatively poorly known (Gagnier \& Wilson 1996), which is likely to further increase the relative influence of tip age priors on its phylogenetic position.

The effect of the stratigraphic sequence operator on the effective tip date prior (Fig. 1C) is desirable. For example, for the younger fossil layer to be close to the maximum age bound for the stratigraphic sequence (e.g. fig. 1B) would require both fossil layers to occur very close together, implying highly heterogeneous sedimentation rates. While this is possible, it is appropriate to assign a low prior probability to this scenario. Further refinements to tip age priors could be added, such as applying non-uniform tip age priors (in addition to the operators), based on the relative position of a layer in a sequence and an assumption of uniform sedimentation rates. The combined effect of the tree prior, operators and tip age priors on the effective prior density on tip dates would need to be analysed by sampling from the prior, as for node age calibrations (Heled \& Drummond 2012).

The MCMC proposals presented here are particularly relevant for specimen-level phylogenetic datasets (e.g. Cau 2017; Tschopp et al. 2015). Even when phylogenetic datasets are not strictly specimen-based, taxa are often scored based on a single specimen or specimens from a single fossil site (as is the case for the phylogenetic dataset utilised here). Simulations have shown that tip dating works best when fossil terminals are assigned ages based on the specimens from which the morphological data were coded (Püschel et al. 2020). The new methods presented here should therefore be widely applicable to phylogenetic analyses of palaeontological data. This includes the use of "clock-less" tip dating to timescale trees, without the use of morphological data (paleotree R package v. 3.3.25 reference manual; Bapst 2012). Correctly handling of fossil ages is also of importance for analyses utilising the unresolved fossilised birth-death model

Peer) reviewing PDF | (2020:02:45500:1:0:NEW 25 Apr 2020) 
212 (Heath et al. 2014), and there is increased appreciation for the need to include adequate fossil 213 samples in such analyses (O’Reilly \& Donoghue 2020).

214

215 We note that these MCMC proposals will not be applicable to all fossil occurrences. Where 216 sedimentation rates are slow, fossil sites can cover millions of years in time; in this case it would 217 be more appropriate to analyse fossils layer by layer using a stratigraphic sequence operator. 218 However, these proposals will be inappropriate when there is considerable reworking of fossils, 219 leading to uncertain relative ages. In cases where morphological data for individual tips are taken 220 from fossils covering a wide stratigraphic range, models that explicitly take stratigraphic ranges 221 (as opposed to uncertainty) into account would be more appropriate (Stadler et al. 2018), although these are not yet implemented in phylogenetic software.

225

226

227

228

229

230

231

\section{2}

233

234

235

236

237

238

239

240

241

242

243

244

245

246

247

248

249

250

251

252

253

\section{Conclusions}

This study introduces new MCMC proposals implemented in BEAST2 designed to deal with stratigraphic age uncertainty of fossils by linking the ages of fossils from the same site, as well as correctly ordering fossil sites within a sequence. When used on an empirical dataset, the use of these new proposals leads to increased precision of site age estimates and minor effects on tree topology. The MCMC proposals presented here should be widely applicable to studies that employ tip dating, particularly for specimen-level datasets.

\section{Acknowledgements}

We thank Remco Bouckaert for advice on BEAST2 and Daniele Silvestro, Eric Gorscak and an anonymous reviewer for comments on the manuscript.

\section{References}

Bapst DW. 2012. paleotree: an R package for paleontological and phylogenetic analyses of evolution. Methods in Ecology and Evolution 3:803-807.

Barido-Sottani J, Aguirre-Fernández G, Hopkins MJ, Stadler T, and Warnock R. 2019. Ignoring stratigraphic age uncertainty leads to erroneous estimates of species divergence times under the fossilized birth-death process. Proceedings of the Royal Society $B$ 286:20190685.

Bouckaert R, Vaughan TG, Barido-Sottani J, Duchêne S, Fourment M, Gavryushkina A, Heled J, Jones G, Kühnert D, De Maio N, Matschiner M, Mendes FK, Müller NF, Ogilvie HA, du Plessis L, Popinga A, Rambaut A, Rasmussen D, Siveroni I, Suchard MA, Wu C-H, Xie D, Zhang C, Stadler T, and Drummond AJ. 2019. BEAST 2.5: An advanced software platform for Bayesian evolutionary analysis. PLoS Computational Biology 15:e1006650.

Burrow C, den Blaauwen J, Newman M, and Davidson R. 2016. The diplacanthid fishes (Acanthodii, Diplacanthiformes, Diplacanthidae) from the Middle Devonian of Scotland. Palaeontologia Electronica 19:1-83.

Cau A. 2017. Specimen-level phylogenetics in paleontology using the Fossilized Birth-Death model with sampled ancestors. PeerJ 5:e3055. 
254

255

256

257

258

259

260

261

262

263

264

265

266

267

268

269

270

271

272

273

274

275

276

277

278

279

280

281

282

283

284

285

286

287

288

289

290

291

292

293

294

295

296

297

298

299

300

301

302

303

304

Coates MI, Finarelli JA, Sansom IJ, Andreev PS, Criswell KE, Tietjen K, Rivers ML, and La Riviere PJ. 2018. An early chondrichthyan and the evolutionary assembly of a shark body plan. Proceedings of the Royal Society B: Biological Sciences 285:20172418.

Drummond AJ, Ho SY, Phillips MJ, and Rambaut A. 2006. Relaxed phylogenetics and dating with confidence. PLoS Biology 4:e88.

Drummond AJ, and Stadler T. 2016. Bayesian phylogenetic estimation of fossil ages. Philosophical Transactions of the Royal Society B 371:20150129.

Gagnier P-Y, and Wilson MV. 1996. Early Devonian acanthodians from northern Canada. Palaeontology 39:241-258.

Gavryushkina A, Heath TA, Ksepka DT, Stadler T, Welch D, and Drummond AJ. 2017.

Bayesian total evidence dating reveals the recent crown radiation of penguins. Systematic Biology 66:57-73.

Gavryushkina A, Welch D, Stadler T, and Drummond AJ. 2014. Bayesian inference of sampled ancestor trees for epidemiology and fossil calibration. PLoS Computational Biology 10:e1003919.

Hanke GF, and Davis SP. 2008. Redescription of the acanthodian Gladiobranchus probaton Bernacsek \& Dineley, 1977, and comments on diplacanthid relationships. Geodiversitas 30:303-330.

Hanke GF, Davis SP, and Wilson MV. 2001. New species of the acanthodian genus Tetanopsyrus from northern Canada, and comments on related taxa. Journal of Vertebrate Paleontology 21:740-753.

Hanke GF, and Wilson MV. 2006. Anatomy of the Early Devonian acanthodian Brochoadmones milesi based on nearly complete body fossils, with comments on the evolution and development of paired fins. Journal of Vertebrate Paleontology 26:526-537.

Hanke GF, and Wilson MVH. 2004. New teleostome fishes and acanthodian systematics. In: Arratia G, Wilson MVH, and Cloutier R, eds. Recent advances in the origin and early radiation of vertebrates. München: Verlag Dr. Friedrich Pfeil, 189-216.

Hanke GF, and Wilson MVH. 2010. The putative stem-group chondrichthyans Kathemacanthus and Seretolepis from the Lower Devonian MOTH locality, Mackenzie Mountains, Canada. In: Elliott DK, Maisey JG, Yu X, and Miao D, eds. Morphology, Phylogeny, and Paleobiogeography of Fossil Fishes, Honoring Meemann Chang. München: Verlag Dr. Friedrich Pfeil, 159-182.

Heath TA, Huelsenbeck JP, and Stadler T. 2014. The fossilized birth-death process for coherent calibration of divergence-time estimates. Proceedings of the National Academy of Sciences 111:E2957-E2966.

Heled J, and Drummond AJ. 2012. Calibrated tree priors for relaxed phylogenetics and divergence time estimation. Systematic Biology 61:138-149.

King B, Qiao T, Lee MS, Zhu M, and Long JA. 2017. Bayesian Morphological Clock Methods Resurrect Placoderm Monophyly and Reveal Rapid Early Evolution in Jawed Vertebrates. Systematic Biology 66:499-516.

Lee MSY, Cau A, Naish D, and Dyke GJ. 2014. Morphological clocks in paleontology, and a mid-Cretaceous origin of crown Aves. Systematic Biology 63:442-449.

Lewis PO. 2001. A likelihood approach to estimating phylogeny from discrete morphological character data. Systematic Biology 50:913-925.

Matzke NJ, and Irmis RB. 2018. Including autapomorphies is important for paleontological tipdating with clocklike data, but not with non-clock data. PeerJ 6:e4553.

Miller RF, Cloutier R, and Turner S. 2003. The oldest articulated chondrichthyan from the Early Devonian period. Nature 425:501-504.

O'Reilly JE, and Donoghue PC. 2018. The efficacy of consensus tree methods for summarizing phylogenetic relationships from a posterior sample of trees estimated from morphological data. Systematic Biology 67:354-362.

Peer) reviewing PDF | (2020:02:45500:1:0:NEW 25 Apr 2020) 
305

306

307

308

309

310

311

312

313

314

315

316

317

318

319

320

321

322

323

324

325

326

327

328

329

330

331

332

333

334

335

336

337

338

339

O'Reilly JE, and Donoghue PCJ. 2020. The effect of fossil sampling on the estimation of divergence times with the fossilized birth-death process. Systematic Biology 69:124138.

Paradis E, Claude J, and Strimmer K. 2004. APE: analyses of phylogenetics and evolution in R language. Bioinformatics 20:289-290.

Püschel HP, O'Reilly JE, Pisani D, and Donoghue PCJ. 2020. The impact of fossil stratigraphic ranges on tip-calibration, and the accuracy and precision of divergence time estimates. Palaeontology 63:67-83.

$\mathrm{R}$ Core Team. 2018. R: A language and environment for statistical computing. $\mathrm{R}$ foundation for Statistical Computing, Vienna, Austria. URL https://www.R-project.org/.

Rambaut A, Suchard MA, Xie D, and Drummond AJ. 2014. Tracer v1.6, Available from http://beast.bio.ed.ac.uk/Tracer.

Revell LJ. 2012. phytools: an R package for phylogenetic comparative biology (and other things). Methods in Ecology and Evolution 3:217-223.

Ronquist F, Klopfstein S, Vilhelmsen L, Schulmeister S, Murray DL, and Rasnitsyn AP. 2012. A total-evidence approach to dating with fossils, applied to the early radiation of the Hymenoptera. Systematic Biology 61:973-999.

Sallam HM, and Seiffert ER. 2016. New phiomorph rodents from the latest Eocene of Egypt, and the impact of Bayesian "clock"-based phylogenetic methods on estimates of basal hystricognath relationships and biochronology. PeerJ 4:e1717.

Stadler T, Gavryushkina A, Warnock RCM, Drummond AJ, and Heath TA. 2018. The fossilized birth-death model for the analysis of stratigraphic range data under different speciation modes. Journal of Theoretical Biology 447:41-55.

Suchard MA, Lemey P, Baele G, Ayres DL, Drummond AJ, and Rambaut A. 2018. Bayesian phylogenetic and phylodynamic data integration using BEAST 1.10. Virus Evolution 4:vey016.

Tschopp E, Mateus O, and Benson RBJ. 2015. A specimen-level phylogenetic analysis and taxonomic revision of Diplodocidae (Dinosauria, Sauropoda). PeerJ 3:e857.

Warren DL, Geneva AJ, and Lanfear R. 2017. RWTY (R We There Yet): An R package for examining convergence of Bayesian phylogenetic analyses. Molecular Biology and Evolution 34:1016-1020.

Wickham H. 2016. ggplot2: elegant graphics for data analysis: Springer.

Yang Z. 1996. Among-site rate variation and its impact on phylogenetic analyses. Trends in Ecology \& Evolution 11:367-372. 


\section{Figure 1}

An MCMC proposal enforcing the correct ordering of fossil sites within a sequence, but allowing overlapping uncertainty bounds

(A-B) The blue lines represented the sampled ages of two fossil sites (light blue: younger site, dark blue:older site). The range of possible values for new proposals at a particular point in the Markov chain (part A and B represent different points in the chain) depends on the current value of the other site in the sequence. Arrows indicate the possible range of new proposals. (C) Implementation of this operator on an empirical dataset leads to non-uniform effective priors on site age (in this case two formations from the Early Devonian of Spitzbergen) . Note that colours are plotted with transparency to show overlap. 

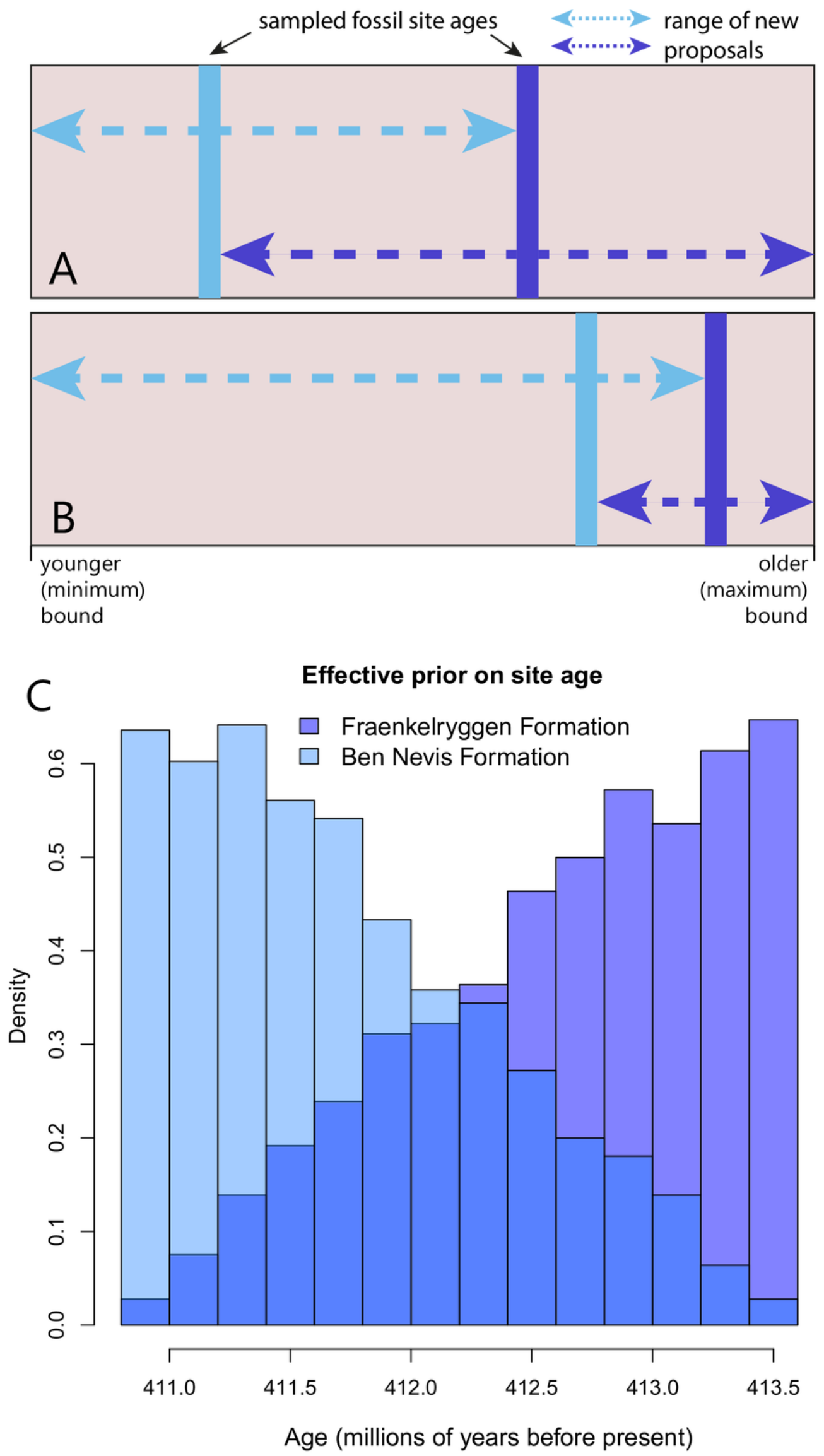


\section{Figure 2}

Linking the tip ages of fossils from the same site leads to increased precision of age estimates and has minor effects on tree topology

(A) 95\% HPD intervals for individual taxa within two fossil sites (light green, Turin Hill taxa; light orange, MOTH taxa), compared with 95\% HPD interval when tip ages within fossil sites are linked (dark green, Turin Hill; dark orange, MOTH). Circles represent median estimates. (B-C) $50 \%$ majority rule cladogram (in part) from the analysis with independent tip ages (B) and with linked tip ages for fossil sites (C). 
A Climatius -

Euthacanthus -

Ischnacanthus-

Mesacanthus -

Parexus -

Turin Hill-

Brochoadmones -

Cassidiceps -

Gladiobranchus -

Kathemacanthus -

Lupopsyrus-

Obtusacanthus -

Promesacanthus -

Tetanopsyrus-

MOTH-

412

414

416

418

Age (millions of years before present)

B Independent tip ages

$50 \%$ majority-rule consensus
C Linked tip ages

$50 \%$ majority-rule consensus
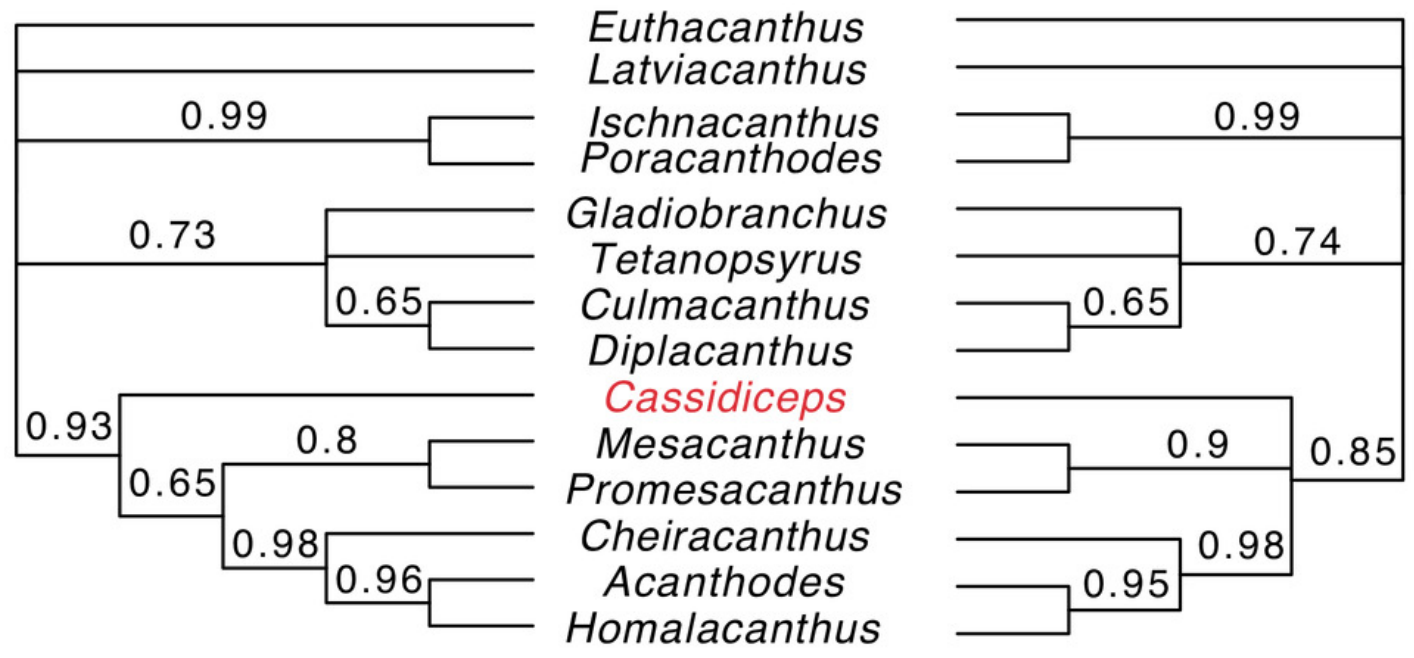